THIRD SESSION

SEPTEMBER 9

Chairman:

M. G. FRACASTORO 


\title{
L'OBSERVATION SPECTROSCOPIQUE DES COUPLES VISUELS
}

\author{
Exposé Introductif
}

\section{J. DOMMANGET}

Observatoire Royal de Belgique, Bruxelles, Belgique

Le concours de diverses techniques d'observation a toujours été profitable au progrès des connaissances astronomiques.

Dans le cas des couples stellaires, l'observation visuelle et l'observation spectroscopique se complètent heureusement: chacune d'elles - lorsqu'elles sont possibles conduit à la connaissance des éléments orbitaux, sauf que la première ne fournit pas l'échelle de l'orbite en unités astronomiques et laisse subsister une certaine indétermination, relative à la position du nœud ascendant, et que la seconde n'apporte aucune information sur la valeur de l'inclinaison et sur celle de l'angle de position de la ligne des nœuds.

La combinaison des informations fournies par les deux techniques d'observation, permet donc de résoudre entièrement et de façon adéquate, le problème de la détermination des éléments orbitaux et cela, même lorsque l'une d'elles ne donne pas tous les renseignements qu'elle est capable de fournir dans le cas théorique idéal.

Il existe sans doute une complémentarité du même genre entre les techniques visuelle et spectroscopique d'une part, et la technique photométrique d'autre part, mais elle ne relève pas du sujet proposé pour la présente séance de travail. Aussi considéreronsnous seulement l'apport relatif de chacune des techniques visuelle et spectroscopique et, plus particulièrement, l'apport de la seconde à la première, considérée ici comme principale.

Mais cet apport est plus important qu'il ne pourrait paraître à la suite de ce que nous venons de rappeler concernant les éléments orbitaux. Aussi vaut-il la peine non seulement de considérer la question sous ses divers aspects en insistant sur son intérêt pour l'astronomie des étoiles doubles, mais aussi d'examiner dans quelle mesure, la technique spectroscopique est applicable au cas - particulier pour elle - des couples visuels.

\section{L'intérêt de l'observation spectroscopique des binaires visuelles}

De façon génerale, l'observation spectroscopique d'un couple stellaire peut fournir à elle seule des renseignements sur:

(a) Les types spectraux et les classes de luminosité de ses composantes;

(b) La parallaxe (spectroscopique);

(c) Les vitesses radiales de son centre de masse et de ses composantes;

(d) Certaines particularités (rotation des composantes sur elles-mêmes, présence d'enveloppes, anomalies spectrales, etc.) auxquelles nous ne nous attarderons pas 
spécialement, puisque leur étude dépend de l'obtention des spectrogrammes et constitue dès lors une sorte de prolongement de celles relevant du premier point cité.

(a) En ce qui concerne les types spectraux et les classes de luminosité de chaque composante des couples stellaires, on sait l'importance qu'ils représentent pour les recherches d'une part sur la relation masse-luminosité dont les binaires visuelles constituent la base principale, et d'autre part sur les particularités du diagramme H-R.

P. Couteau, dans un exposé introductif présenté lors du Colloque UAI tenu à Bruxelles sur les étoiles doubles (1967) remarquait, à juste titre, que "la finesse de la relation masse-luminosité dépend maintenant des progrès sur la correction bolométrique" (p. 185), laquelle est, comme le rappelle l'auteur, "liée d'une part à la magnitude bolométrique ... et d'autre part, à la magnitude mesurée par les récepteurs". Mais il ne faudrait pas en conclure que le problème général est devenu essentiellement photométrique car, en ce qui concerne l'établissement de cette relation, une quarantaine de couples seulement sont actuellement disponibles, le choix s'effectuant sans doute pour des raisons liées à la qualité des orbites, mais aussi parce que bien souvent, d'autres données fondamentales manquent ou sont insuffisamment précises, en particulier les données spectroscopiques individuelles sur les somposantes.

Par ailleurs, l'application de la relation masse-luminosité à un plus grand nombre de couples à orbites connues, par exemple en vue de la détermination de leurs parallaxes dynamiques et en vue de recherches statistiques diverses ayant trait généralement à l'évolution stellaire et même indirectement à l'évolution galactique, implique également cette connaissance des spectres individuels des composantes. Or, nous remarquerons, à titre d'information, que, d'après le Catalogue d'orbites de Worley (1963), pour 48 seulement des 536 couples qu'il contient, les spectres des composantes sont connus, soit pour quelque $9 \%$ !

Quant à la distribution des binaires - orbitales ou non - dans le diagramme H-R, il est évident que son étude sera d'autant plus intéressante que l'on disposera de meilleures informations sur les spectres des astres considérés. Ici aussi, la connaissance des spectres "résultants" constitue un minimum nettement insuffisant pour situer chaque couple correctement dans le diagramme et tirer des conclusions valables concernant son évolution possible.

P. Couteau, dans l'exposé cité plus haut, faisait remarquer que les nombreuses études sur la distribution des composantes des étoiles doubles dans le diagramme H-R n'ont pratiquement porté que sur des ensembles de binaires formées de composantes écartées et dès lors généralement non orbitales. Ce qui lui fait écrire (p. 187): "Ce ne sont pas toujours les mêmes étoiles qui servent d'étalon à la relation masse-luminosité et au diagramme H-R".

Notons encore que, dans son rapport de Président de la Commission No. 29 à l'Assemblée Générale de l'UAI à Moscou en 1958, J. L. Greenstein écrivait: "The spectra of visual binaries are much neglected; each binary is a miniature cluster and because of their large number, they should permit refinement of concepts of stellar evolution hitherto based on galactic or globular systems. The frequency of spectro- 
scopic and visual binaries as a function of population type is not known" (Transactions $U A I$ X, p. 446).

Depuis lors, des progrès ont été réalisés dans cette voie, mais un travail important reste à faire.

(b) Au sujet des parallaxes, il faut noter que si les spectrogrammes à grandes dispersions permettent des déterminations de parallaxes, dites spectroscopiques par l'évaluation des magnitudes absolues, ceux obtenus à l'aide de prismes-objectifs conduisent généralement à la connaissance non seulement des types spectraux, mais aussi des classes de luminosité, ce qui permet d'assurer également de bonnes valeurs pour les magnitudes absolues et dès lors de conclure indirectement aux valeurs des parallaxes.

En l'absence des techniques spectroscopiques, on ne disposerait d'ailleurs que de très peu de renseignements valables sur les distances qui nous séparent des couples éloignés.

(c) La détermination des vitesses radiales à partir de spectrogrammes à grandes dispersions et de clichés obtenus aux prismes-objectifs de Ch. Fehrenbach, est pour les couples visuels d'un intérêt multiple. On y distingue les aspects suivants:

\subsection{La CONNAISSANCE DES VITESSES SPATIALES}

obtenues par composition des vitesses radiales, des mouvements propres et des parallaxes. Son importance est évidente pour l'étude de la participation des couples aux courants stellaires dans le voisinage du Soleil et pour l'étude de leur formation et de leur évolution (Lippincott, 1967) en liaison avec les caractéristiques galactiques locales.

La valeur de ces recherches dépend fondamentalement de la précision des données. A ce sujet, il peut être intéressant de consulter le diagramme de la figure 1, établi à partir du Catalogue des vitesses radiales de Wilson (1953) et donnant, en fonction de la classe spectrale, la fréquence des $V R$ appartenant aux diverses catégories de l'échelle de précision considérée par l'auteur. En établissant ce diagramme, nous n'avons pas constaté de différence significative entre les étoiles de la série principale et les géantes.

Or, comparée à la précision des valeurs des composantes tangentielles des vitesses spatiales, celle des $V R$ paraît l'emporter dans l'ensemble. En effet, la vitesse tangentielle $T$, est donnée par

$$
T_{\mathrm{km} / \mathrm{s}}=4,74{ }_{\pi^{\prime \prime}}^{\mu^{\prime \prime}},
$$

où $\mu^{\prime \prime}$ est le mouvement propre annuel. On voit immédiatement que dans les cas les plus favorables où d'une part les binaires sont proches $\left(\pi^{\prime \prime}>00^{\prime \prime} 100\right)$ et les parallaxes, connues à un peu mieux de $10 \%$ et où d'autre part, les mouvements propres sont connus avec une précision au moins égale, les erreurs affectant les valeurs déduites de $T$ sont voisines de $10 \%$ au moins, ce qui, pour des valeurs de $T$ de l'ordre de $10 \mathrm{~km} / \mathrm{s}$ conduit à une précision à peine comparable aux meilleures déterminations de $V R$ (classe a) et pour des valeurs de $T$ de $50 \mathrm{~km} / \mathrm{s}$, entraîne une assimilation aux $V R$ de la classe $d$.

Mais ceci n'est que la transposition au domaine des étoiles doubles, de ce qui est 
bien connu pour les étoiles simples. Il semblait toutefois utile de rappeler que les données spectroscopiques n'ont ici rien à envier, dans l'ensemble, a ux données astrométriques.

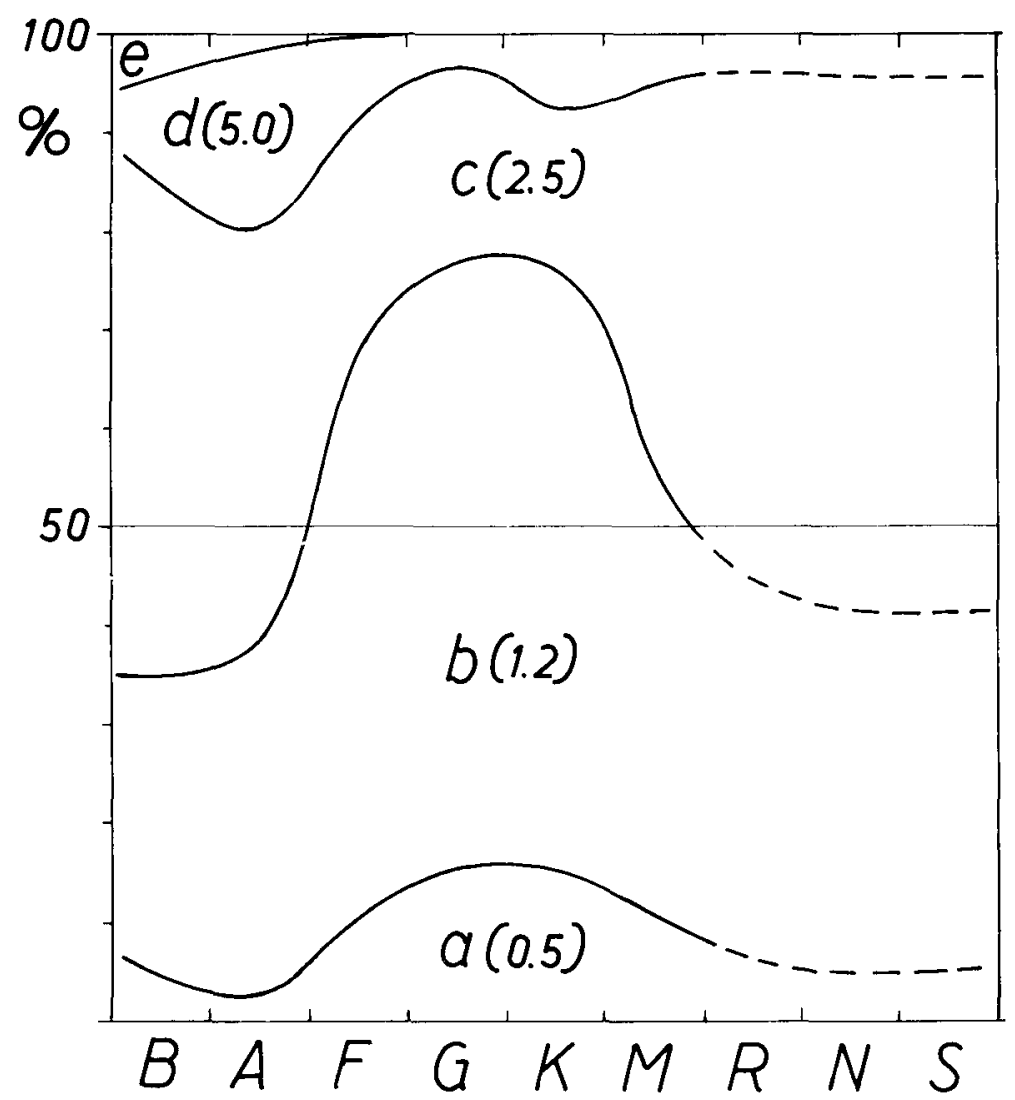

Fig. 1. Fréquences des $V R$ de diverses précisions en fonction de la classe spectrale. Les classes $a, b, c, d$ et $e$ sont celles utilisées par R. E. Wilson dans son Catalogue de Vitesses Radiales (1953).

Les valeurs indiquées entre parenthèses sont les erreurs probables correspondantes, en $\mathrm{km} / \mathrm{s}$.

C'est une des raisons pour lesquelles seulement 30 à 50 couples peuvent être retenus actuellement dans les recherches portant par exemple sur l'orientation des plans orbitaux relativement aux vitesses spatiales des binaires (Lippincott, 1967).

\subsection{LA CONNAISSANCE DES PARALLAXES}

par une voie indirecte, mais particulièrement intéressante pour les couples orbitaux éloignés $\left(\pi^{\prime \prime}<0.020\right)$, car le procédé, sous l'angle de la précision, semble être alors compétitif avec tous les autres connus. En effet, la parallaxe est donnée par la relation

$$
\pi^{\prime \prime}=29,76 \frac{a^{\prime \prime} \sin i}{P K \sqrt{1-e^{2}},}
$$

où $K$ est la demi-amplitude en $\mathrm{km} / \mathrm{s}$, de l'orbite spectroscopique. 
Si $V r$ est la vitesse radiale relative des composantes, à un instant donné, on a

$$
V r=K[e \cos \omega+\cos (v+\omega)]
$$

et (1) s'écrit

$$
\pi^{\prime \prime}=29,76 \stackrel{a^{\prime \prime}}{P W V r},
$$

où

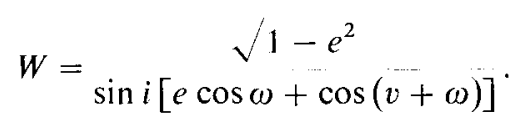

Quand l'orbite visuelle est connue, l'expression (2) peut s'appliquer à chacune des déterminations de $V r$. La considération de plusieurs déterminations successives permet de chiffrer l'imprécision sur $\pi^{\prime \prime}$, due aux erreurs affectant les valeurs de $V r$. En vue de connaître l'erreur totale sur les valeurs déduites pour $\pi^{\prime \prime}$, il serait intéressant d'étudier indépendamment, l'erreur affectant l'expression $a^{\prime \prime} \mid P W$, en considérant des orbites successives d'un même couple, comme nous l'avons fait pour $\log a^{\prime \prime}, \log P$ et pour l'expression $3 \log a^{\prime \prime}-2 \log P$, et dont nous parlerons à la prochaine séance (voir p. 137).

Mais on remarquera dès maintenant que $10 \%$ d'erreur sur l'expression $a^{\prime \prime} \mid P W$ ou sur la valeur observée de $V r$, entraîne une erreur du même ordre sur $\pi^{\prime \prime}$, ce qui peut être considéré comme très acceptable lorsque $\pi^{\prime \prime}$ est petit, inférieur à 0.020 par exemple.

Quant à la formule (1), elle n'est applicable que lorsque l'orbite spectroscopique toute entière est également connue c'est-à-dire $K$, en plus de l'orbite visuelle. Elle conduit toutefois à des valeurs de $\pi^{\prime \prime}$ intrinsèquement plus précises puisque la valeur de $K$ est basée sur l'ensemble des observations de $V r$ ayant conduit à la détermination orbitale.

On ne connaît malheureusement qu'une application concluante de ces principes: il s'agit de l'étoile $\alpha$ Centauri. Mais malgré les difficultés - dont nous parlerons plus loin - d'obtenir les vitesses radiales des deux composantes d'un couple visuel, il semble qu'une préparation convenable des programmes d'observation devrait permettre, en quelques années, le traitement de plusieurs cas de ce genre.

Remarquons encore que les parallaxes déduites par cette voie, sont des parallaxes absolues.

\subsection{LA CONNAISSANCE DES MASSES TOTALES ET INDIVIDUELLES}

dont l'importance n'est pas à rappeler. Notons que, de la relation

$$
\mathfrak{M}_{A B}=\begin{aligned}
& a^{\prime \prime 3} \\
& \pi^{\prime \prime 3} P^{2}
\end{aligned}
$$

on déduit, en y remplaçant $\pi^{\prime \prime}$, soit par son expression (1), soit par son expression (2),

$$
\mathfrak{M}_{A B}=37,93 \times 10^{-6} K^{3} \frac{P \cos ^{3} \phi}{\sin ^{3} i}, \quad(\sin \phi=e)
$$

et

$$
\mathfrak{M}_{A B}=37,93 \times 10^{-6} P W^{3} V r^{3} .
$$


On remarquera que le demi-grand axe $a^{\prime \prime}$ n'apparaît pas dans ces deux dernières expressions et qu'il s'y trouve remplacé par la vitesse radiale relative $V r$, laquelle se présente soit sous la forme de la demi-amplitude $K$ du phénomène spectroscopique (relation (4)), soit sous sa forme propre (relation (5)).

Ici aussi, il y aurait intérêt à connaître l'importance des erreurs susceptibles d'affecter l'expression $P W^{3}$, en considérant des orbites successives d'un même couple, afin de déterminer finalement l'erreur totale affectant les valeurs déduites pour la masse totale, par l'emploi de (5).

La remarque faite quant à l'ampleur du domaine d'application en ce qui concerne la connaissance des parallaxes, est valable, bien entendu, dans le cas présent.

Pour la détermination des masses individuelles, il est évidemment nécessaire de connaître les demi-amplitudes $K_{A}$ et $K_{B}$, des orbites spectroscopiques des composantes ou du moins, la vitesse radiale du centre de masse, à moins que des observations méridiennes ou astrométriques n'aient permis de situer ce centre précédemment.

\subsection{LA LEVÉE D'AMBIGUÏTÉ DE POSITION DES NCEUDS ASCENDANTS}

indispensable à l'étude de l'orientation des plans orbitaux. Actuellement, sur les quelque 600 couples dont l'orbite est connue, la levée d'ambiguïté de position des nœuds ascendants n'a été possible que pour environ 50 d'entre eux (Batten, 1967; Dommanget, 1969). Il semble cependant que l'on pourrait assez aisément doubler cette proportion en quelques années d'observation des $\operatorname{Vr}$ (Dommanget, 1967).

Il est à noter que chaque cas n'exige pas nécessairement la connaissance des vitesses

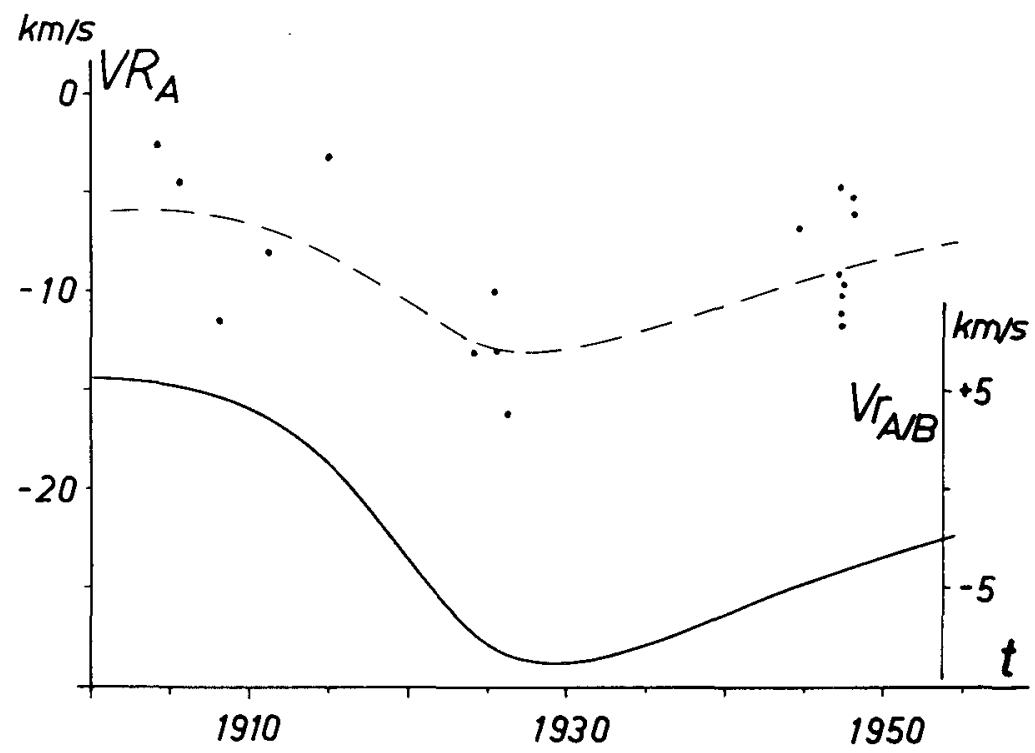

Fig. 2. Graphique permettant la comparaison de la courbe des vitesses radiales relatives de $A$ par rapport à $B$ (on calcule généralement celle de $B$ par rapport à $A$ (Dommanget et Nys, 1967)) et des observations de la vitesse radiale de la composante principale $A$ du couple ADS 8739 (12564 N 5654). 
radiales des deux composantes. Au contraire, dans la plupart des cas, l'observation régulière de la principale permet d'assurer la forme de la courbe des vitesses radiales et de procéder ainsi au choix de celle des deux solutions offertes par l'orbite visuelle. La Figure 2 illustre ce point par un cas particulièrement évident, où l'on dispose d'observations réparties sur un assez long intervalle de temps. Mais assez souvent un choix convenable des époques d'observation permet de ne s'intéresser qu'à un intervalle de temps relativement court et de conclure avec tout autant de certitude (Figure 3 ).

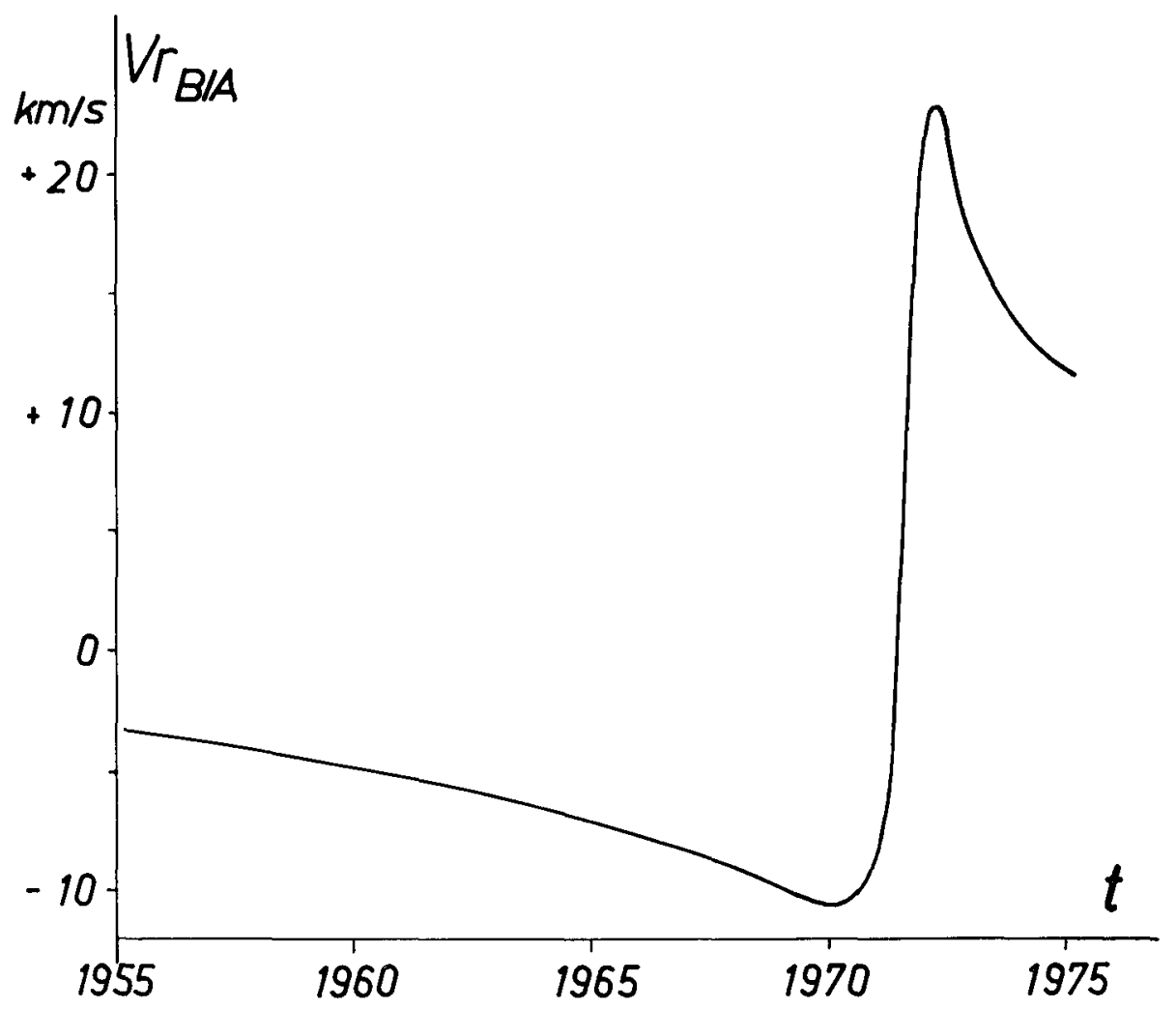

Fig. 3. Courbe de $V r$ pour le couple ADS 15176 (21344 S 0030) AB, d'après notre catalogue d'éphéméride de $\operatorname{Vr}$ (Dommanget et Nys, 1967). L'observation spectroscopique régulière au cours de la période 1970-1975 devrait permettre de savoir si la courbe des $V r$ tourne sa concavité (en 1970) vers les valeurs positives ou vers les valeurs négatives. Passé cette époque, il faudra attendre sans doute longtemps ( $P=48,7$ ans) avant de pouvoir retrouver les mêmes conditions permettant de traiter ce cas aisément.

\subsection{LA DISCRIMINATION ENTRE ORBITES VISUELLES DISTINCTES MAIS ÉGALEMENT POSSIBLES D'UN MÊME COUPLE}

D’assez nombreux couples serrés et ne présentant pas de différence d'éclat notable entre leurs composantes, conduisent à deux solutions orbitales distinctes, par suite d'une confusion se présentant entre l'étoile considérée primitivement comme principale et son compagnon, après leur resserrement maximal, c'est-à-dire après une période 
plus ou moins longue au cours de laquelle le couple n'est plus visuellement observable.

Deux solutions (parfois quatre) sont alors proposées et conduisent à des courbes de vitesses radiales toujours forts différentes les unes des autres. Des observations spectroscopiques faites à l'époque la plus opportune, peuvent alors permettre de reconnaître la seule solution valable. La Figure 4 illustre un tel cas. Cependant le problème est

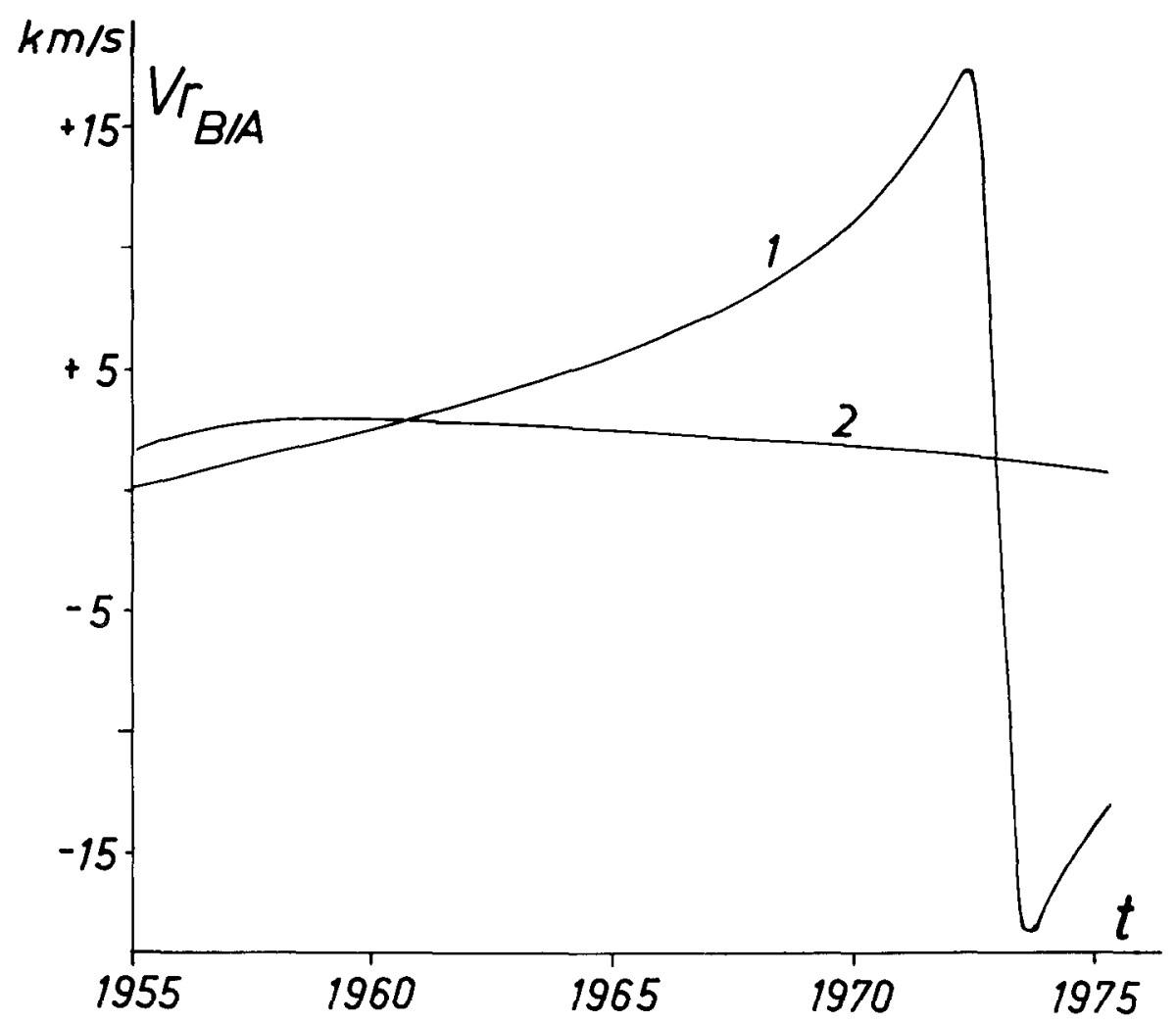

Fig. 4. Courbes de vitesses radiales calculées (Dommanget et Nys, 1967) pour le couple ADS 9195 (14122 N 3400) respectivement avec les éléments orbitaux de P. Couteau (1) et de P. Baize (2).

parfois délicat. Nous rappellerons à cette occasion le cas de ADS 1123 que nous avons détaillé ailleurs (1967, p. 16).

Il est évident que l'observation des vitesses radiales peut également servir au choix entre diverses orbites également représentatives du matériel d'observation visuel, même lorsqu'il n'y a pas d'indétermination de quadrant.

\subsection{L'APPLICATION DE CRITÈRES D'OPTICITÉ}

Certains couples dont les composantes ne présentent pas de mouvement relatif appréciable, témoignent parfois de vitesses radiales relatives importantes, supérieures à la vitesse parabolique. Sur la base de cette remarque, on peut établir des critères 
d'opticité, la relation trouvée (Dommanget, 1960) étant

$$
0,44 \log \pi_{i}^{\prime}=-3,249+2 \log V r+\log \varrho+0,1117 m_{A}^{\prime}-\log \mu,
$$

où $V r$ est la vitesse radiale relative des composantes; $\varrho$, leur distance de séparation; $m_{A}^{\prime}$, la magnitude apparente visuelle de l'étoile principale corrigée de la correction bolométrique (P. Baize); $\log \mu$, une fonction de la différence des magnitudes; $\pi_{i}^{\prime}$, une limite de la parallaxe à laquelle la parallaxe observée ne peut être inférieure sans que le couple soit optique.

Cette relation n'est valable que si les composantes satisfont à la relation masseluminosité. Appliquée à quelque 350 couples de l'ADS, 21 ont été reconnus optiques.

L'intérêt des critères d'opticité basés sur les vitesses radiales réside dans le fait qu'ils sont applicables - en principe - dès la connaissance d'une première valeur de celle-ci tandis que ceux basés sur les mouvements apparents exigent au moins deux observations de position effectuées à un intervalle de temps suffisamment grand (20, 50 voire 100 ans) pour permettre la déduction de la vitesse apparente relative toujours assez faible, même dans les cas les plus favorables. Cette remarque concerne surtout les couples assez éloignées.

\section{Les problèmes liés à l'observation spectroscopique des couples visuels}

Si, théoriquement, l'énoncé que nous venons de faire des divers domaines d'application des techniques spectroscopiques dans l'astronomie des étoiles doubles visuelles, est vaste et prometteur, il apparaît cependant que, du point de vue pratique, des difficultés d'observation nombreuses et variables d'un couple à l'autre affectent sérieusement l'étendue du domaine exploitable. La situation n'est pas dramatique mais on ne peut ignorer ces difficultés.

Les facteurs intervenant dans la limitation des possibilités d'observation spectroscopique des couples stellaires sont les magnitudes et les distances de séparation de leurs composantes, mais aussi l'importance de leurs vitesses radiales relatives.

\subsection{L'ÉCLAT DE CHACUNE DES COMPOSANTES}

Si l'on considère les composantes des étoiles doubles comme des étoiles simples, l'emploi de prismes-objectifs et l'obtention de spectrogrammes à faible et moyenne dispersions permettent de fournir les informations essentielles sur la classification spectrale de tous les couples connus (Index Catalogue, 1963), leurs magnitudes ne dépassant généralement pas la $15 \mathrm{e}$.

Par contre, si, dans les mêmes conditions, on s'intéresse aux spectrogrammes à grandes dispersions (vitesses radiales, particularités spectrales diverses), les étoiles faibles ne sont plus atteintes. En considérant les étoiles contenues dans le Catalogue de vitesses radiales de Wilson (1953), on trouve en effet une limite supérieure située entre la 9e et la 12e magnitude environ suivant le type spectral, et distincte pour les étoiles de la séquence principale et les géantes, comme l'indique le graphique de la Figure 5.

Cette limite tient compte des possibilités globales de tous les observatoires du monde 
entier et représente donc un optimum difficilement atteint. Mais il ne faut pas perdre de vue que cette limite est celle d'un catalogue établi il y a plus de 15 ans. Elle est d'ailleurs plus élevée par exemple pour les vitesses radiales obtenues au prismeobjectif de Fehrenbach (1967), encore inconnu à l'époque.

Ces diverses remarques ne sont cependant valables que pour les étoiles simples ou

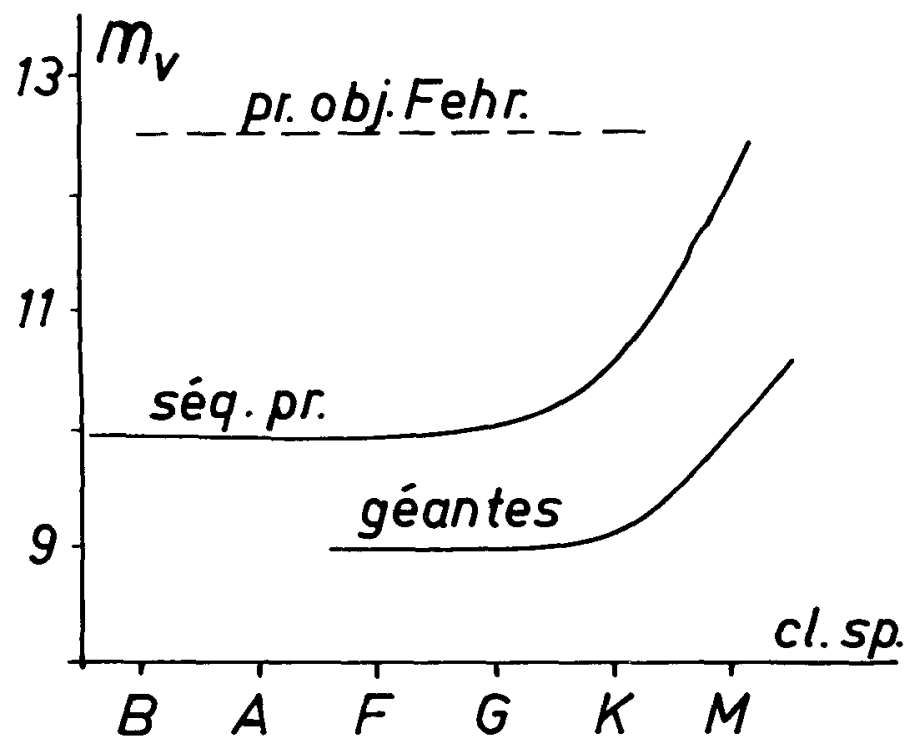

Fig. 5. Magnitude limite pour l'obtention de spectrogrammes à grandes dispersions destinés à la détermination des vitesses radiales à l'aide des instruments les plus puissants, et du prisme-objectif de Fehrenbach (1967).

pour les composantes des binaires considérées individuellement. En fait, la situation est plus complexe pour les binaires visuelles en général, par suite du voisinage prononcé de leurs composantes sur le ciel.

\subsection{LA DISTANCE DE SÉPARATION}

Si la distance de séparation des composantes est grande, plus de $3^{\prime \prime}$ par exemple, on a affaire au cas traité ci-dessus ou chacune d'elles peut être considérée comme une étoile simple, puisqu'elles sont observables séparément. Et cela d'autant plus que cette séparation est grande, surtout lorsqu'il s'agit d'observations au prisme-objectif.

On remarquera toutefois que les couples les plus intéressants, les couples orbitaux, ont des distances généralement inférieures à $3^{\prime \prime}$ et ne peuvent être traités comme indiqué jusqu'à présent.

En effet, si la distance de séparation est petite, inférieure à $l^{\prime \prime}$ pour préciser les idées, il n'est plus possible généralement de séparer spectroscopiquement les deux étoiles et l'on ne peut observer que le spectre 'résultant' donné par l'ensemble de l'énergie lumineuse émise par la binaire.

Deux cas se présentent alors suivant l'importance de la différence d'éclat des étoiles 
composant le couple. Si cette différence est relativement importante (plus de 2 magnitudes), le spectre résultant se confond avec le spectre de l'étoile principale et, du point de vue des possibilités spectroscopiques, l'étoile observée apparaît comme une étoile simple. On peut alors connaitre son type spectral et sa classe de luminosité et, si sa magnitude ne dépasse pas les limites indiquées à la Figure 5, procéder à la mesure de sa vitesse radiale. Toutefois, il sera impossible d'obtenir un renseignement quelconque - par voie spectroscopique - sur la nature physique du compagnon et sur sa vitesse radiale.

Malgré cela, on pourra, dans une certaine mesure, traiter plusieurs problèmes relevant de la connaissance des vitesses radiales, à condition toutefois de disposer d'observations en nombre suffisant et bien réparties dans le temps, pour assurer certaines particularités de la courbe des $V r$. C'est le cas des étoiles doubles spectroscopiques à un seul spectre observable et d'un certain nombre de couples visuels.

Remarquons qu'une différence de magnitude très grande peut correspondre à un rapport de masse extrême et dès lors, dans le cas des couples visuels, - pour lesquels les valeurs de $K$ sont toujours relativement faibles - à des fluctuations orbitales de la vitesse radjale de la principale, trop petites pour permettre de tirer une quelconque conclusion de son observation.

Par contre, si la différence d'éclat des deux étoiles est relativement faible (moins de deux magnitudes), le spectre global est un spectre résultant typique constitué de la fusion des deux spectres individuels dont il n'est possible de tirer des renseignements que dans certaines circonstances, comme nous allons le voir.

\subsection{LA VITESSE RADIALE RELATIVE}

Si les vitesses radiales relatives sont importantes, la séparation des raies appartenant aux deux composantes se fait assez aisément et permet de reconnaître les deux spectres tant en ce qui concerne leurs particularités propres qu'en ce qui concerne la mesure des vitesses radiales relatives. C'est dans cette catégorie d'étoiles doubles que l'on trouve toutes les doubles spectroscopiques à deux spectres observables ainsi que quelques couples visuels.

Mais si finalement, en plus d'une distance de séparation et d'une différence de magnitude petite, la différence des vitesses radiales est faible, le spectre résultant implique une étude délicate ne conduisant pas toujours à un résultat valable.

C'est malheureusement le cas d'un assez grand nombre d'étoiles doubles visuelles, puisque par suite de périodes relativement grandes, les demi-amplitudes des courbes de $V r$ sont généralement faibles. Nous avons trouvé (1967) parmi les couples à orbites connues, les fréquences suivantes:

$\begin{array}{ll}20 \% \text { pour lesquels } & K<5 \mathrm{~km} / \mathrm{s} \\ 47 & 5 \leqslant K<10 \\ 16 & 10 \leqslant K<15 \\ 9 & 15 \leqslant K<20 \\ 8 & 20 \leqslant K<25 .\end{array}$


alors que pour les couples spectroscopiques à deux spectres observables, la statistique montre que toutes les valeurs de $K$ sont, à quelques rares exceptions près, supérieures à $30 \mathrm{~km} / \mathrm{s}$ et se répartissent très uniformément jusqu'à plusieurs centaines de $\mathrm{km} / \mathrm{s}$.

Mais la situation est moins sombre qu'elle pourrait paraître par le simple rapprochement de ces deux remarques. En fait, pour de nombreux couples visuels, le jeu des divers éléments orbitaux (surtout l'excentricité) conduit à des valeurs maximales de $\mathrm{Vr}$ nettement supérieures à $K$, mais bien entendu, toujours inférieures à $2 K$. Par ailleurs, la connaissance d'orbites spectroscopiques de couples à deux spectres observables dont la valeur atteint le minimum de $30 \mathrm{~km} / \mathrm{s}$, signifie que les observations de $V r$ sont encore possibles un peu en dessous de cette limite. On sait d'ailleurs que des spectrogrammes obtenus dans de bonnes conditions, avec une dispersion de $40 \AA / \mathrm{mm}$ par exemple, permettent de mesurer assez aisément des $V r$ de quelque $20 \mathrm{~km} / \mathrm{s}$ et de descendre, dans les conditions les meilleures d'observation et de dispersion, à environ $10 \mathrm{~km} / \mathrm{s}$. Notons encore que des procédés photoélectriques d'observation permettent de séparer les composantes des raies de spectres résultants à partir de vitesses radiales atteignant seulement 6 à $7 \mathrm{~km} / \mathrm{s}$ (Griffin, 1967) par exemple pour des spectres de type $K$.

Il est donc évident que l'observation des vitesses radiales relatives des étoiles doubles visuelles présentant deux spectres observables est possible dans bien des cas, mais qu'elle réclame l'emploi des instruments les plus puissants et des techniques les plus élaborées.

\section{Problèmes liés à l'exploitation des observations spectroscopiques}

Bien des données spectroscopiques issues de l'observation et indispensables pour résoudre les questions évoquées plus haut en ce qui concerne les étoiles doubles, sont difficilement accessibles, par suite d'une grande dispersion dans les publications diverses, ou encore, sont publiées incomplètement.

\subsection{Types SPECTRAuX et Classes de luminosité}

Ces résultats d'observation sont dispersés dans les publications spécialisées. Il est heureux que l'on puisse s'adresser actuellement à plusieurs centres d'information installées aux observatoires Perkins, Yerkes, de la Plata et de Michigan (Ann Arbor) (Transactions $U A I \mathbf{1 1} \mathbf{A}$, p. 335 et 12 B, p. 294), aptes à fournir les renseignements spectroscopiques souhaités.

Il n'empêche qu'il subsiste un problème d'homogénéité, difficile à traiter pour un non spécialiste. Seul un catalogue général pourrait résoudre ce problème, mais il réclamerait un travail de très grande envergure. Le catalogue de Jaschek (1964) répond en partie à cette nécessité en réunissant la plupart des étoiles (15.000) dont on connait le type spectral mais également la classe de luminosité dans le système de classification de Morgan-Keenan.

Il faut noter que l'Index Catalogue ne donne généralement que les spectres des couples tels qu'ils figurent dans le catalogue de Harvard. 


\subsection{Parallaxes SPeCtroscopiques}

C'est dans ce domaine que le plus d'homogénéité semblait exister peu après la publication en 1935, par F. Schlesinger, de son Catalogue de parallaxes trigonométriques et spectroscopiques. Un nouveau catalogue et des compléments ont été publiés depuis, pour les parallaxes trigonométriques, mais rien n'a été fait du côté des parallaxes spectroscopiques. La question a toutefois été débattue lors de l'Assemblée Générale de l'UAI à Prague en 1967 et un Groupe de Travail a été constitué avec pour mission d'établir un rapport devant permettre de juger de l'opportunité de réaliser et de publier un tel catalogue.

\subsection{VITESSES RADIALES}

Le catalogue des vitesses radiales de Wilson (1953) constitue une cuvre denvergure mais elle ne peut servir qu'à la connaissance des vitesses moyennes déduites de toutes les mesures faites pour les couples comme pour les étoiles simples et le cas échéant pour celle des composantes individuellement.

Mais il y manque la plupart des éléments de référence bibliographique nécessaires pour retrouver les observations individuelles et leurs époques dont l'importance est essentielle dans le domaine des étoiles doubles. Conscient de cette lacune, Abt (1967) a entrepris l'établissement d'un catalogue bibliographique de toutes les références concernant les vitesses radiales.

Par ailleurs, même dans les publications originales, il n'est pas rare de ne trouver aucune indication sur les époques d'observation. Il en va ainsi pour la plupart des observations du Mont Wilson et pour certaines de l'Observatoire Yerkes. Ici également, Abt (1967) a entrepris de combler cette lacune en préparant un catalogue de toutes les observations de $V r$ faites au Mont Wilson, en ayant soin de les compléter de leurs époques respectives.

Je tiens à saisir cette occasion pour rendre hommage à cette double initiative personnelle de la plus haute importance pour le sujet qui nous préoccupe.

Cependant, il est évident qu'une part des lacunes existant dans les informations spectroscopiques dont nous désirerions disposer, trouve son origine chez les astronomes s'occupant des étoiles doubles visuelles qui n'informent que trop rarement les spécialistes de la spectroscopie, de leurs problèmes. Car si l'on désire voir prendre de plus en plus de spectrogrammes des couples visuels qui nous intéressent, il est avant tout de notre devoir de fournir aux observateurs, toutes les données dont ils ont besoin pour l'établissement de leurs programmes. Aussi, est-ce dans cet esprit que nous avons édité un Catalogue d'éphémérides de vitesses radiales relatives, pour tous les couples dont les orbites sont connues (Dommanget et Nys, 1967) en ayant soin d'y donner toutes les informations nécessaires à l'observation.

\section{Conclusion}

On a vu l'importance et la variété des renseignements que l'on peut tirer de l'observation 
spectroscopique des couples visuels. Un important chemin a déjà été parcouru dans cette voie mais, bien que celle-ci soit parsemée de difficultés diverses, il semble que l'on n'ait pas encore exploité toutes les ressources dont on dispose.

Un effort dans ce sens est d'autant plus indiqué que les étoiles doubles visuelles, à l'encontre des étoiles doubles spectroscopiques aux composantes plus serrées, constituent des exemples parfaits du problème des deux corps: pas d'échanges de masses entre les composantes, pas de marées les déformant, etc. Ils constituent donc un ensemble d'objets susceptibles de présenter par exemple certains phénomènes d'évolution dans toute leur pureté.

Alors qu'il est évidemment impossible de demander aux spectroscopistes d'observer individuellement les 64000 couples de l'Index Catalogue (1963), il paraît raisonnable par contre de les intéresser à l'observation des quelques centaines de couples à orbites connues, en vue de réunir à leur sujet, tous les renseignements que l'on peut attendre de la spectroscopie. Ceci a été fait à plusieurs reprises et il faut se féliciter de l'accueil très favorable recueilli auprès de ceux qui pourraient apporter leur aide à cette œuvre, et cela, particulièrement lors du Symposium de l'UAI qui s'est tenu à Toronto en 1966, sur la détermination des vitesses radiales.

Mais il nous semble néanmoins, au point où en sont ces questions actuellement que des progrès supplémentaires substantiels ne seront réalisés que par une collaboration efficace entre les membres des Commissions 30 et 26 de l'UAI et en particulier par une mise en place effective du groupe de travail proposée lors de la XIIIme Assemblée Générale de l'Union à Prague en 1967.

Puisse ce Colloque conduire à la réalisation de ce væu.

\section{Bibliographie}

Abt, H. A.: 1967, 'Two Radial Velocity Catalogues', Proc. IAU Symp. No. 30, Toronto, p. 55.

Batten, A. H.: 1967, 'The Orientation of the Orbital Planes of Fifty-two Visual Binary Systems', Proc. IAU Symp. No. 30, Toronto, p. 199.

Couteau, P.: 1967, 'Relation masse-luminosité et diagramme H-R dans le cas des binaires', - Colloque U.A.I. On the Evolution of Double Stars; Comm. Obs. Roy. Belgique, Série B, No. 17, p. 181.

Dommanget, J.: 1960, 'Second critère de non-périodicité du mouvement relatif d'un couple stellaire visuel', Bull. Astron. 23, fasc. 2, p. 101 ; Comm. Obs. Roy. Belgique, No. 174.

Dommanget, J.: 1967, 'L'intérêt de l'observation des vitesses radiales des composantes des couples stellaires visuels et la parution prochaine d'un catalogue d'éphémérides', Proc. IAU Symp. No. 30 Toronto, p. 195.

Dommanget, J.: 1969, 'La cinématique des couples stellaires visuels dans le voisinage du Soleil', Bull. Astron. Obs. Roy. Belgique 6, p. 246.

Dommanget, J. et Nys, O.: 1967, 'Catalogue d'éphémérides des vitesses radiales relatives des composantes des étoiles doubles visuelles dont l'orbite est connue', Comm. Obs. Roy. Belgique, Série B, No. 15.

Fehrenbach, Ch.: 1967, 'Les mesures de vitesses radiales au Prisme-Objectif', Proc. IAU Symp. No. 30, Toronto, p. 65.

Griffin, R. F.: 1967, 'Photoelectric Measurement of Radial Velocities', Proc. IAU Symp. No. 30, Toronto, p. 3 .

Jaschek, C. O. R.: 1964, 'Catalogue of Stellar Spectra Classified in the Morgan-Keenan System', Obs. Astron. Univ. Nacional La Plata, Serie Astrónomico 28 (2).

Lippincott, S. L.: 1967, 'Orbital Planes and Space Motions of Visual Binaries', Colloque U.A.I. On the Evolution of Double Stars, Com. Obs. Roy. Belgique, Série B, No. 17, p. 68. 
Wilson, R. E.: 1953, General Catalogue of Stellar Radial Velocities, Carnegie Institution of Washington, Publication 601.

Worley, C. E.: 1963, A Catalog of Visual Binary Orbits, Publ. of the U.S. Naval Observatory, 2d Series, 18, part. III.

\section{Discussion}

Muller: À l'Assemblée Générale de l'UAI en 1958, il y a eu une démarche de Muller et Dommanget, à la Commission 30 pour poser le problème; il faut tout de suite:

(1) savoir qui peut fournir les vitesses radiales nécessaires et est disposé à faire les observations;

(2) faire savoir par Dommanget et Nys les quelques couples dont l'observation est actuellement intéressante.

Strand: For how many visual binaries have spectroscopic orbits been published during the past ten years?

Dommanget: I have no record. Anyway, spectroscopic orbits are not especially needed. We are more interested in radial velocity data during the most interesting parts of the orbits.

Batten: This work requires time to complete, and not many orbits were published in the last years. Perhaps about ten systems will be reported on today.

Heintz: Opposing Dommanget's opinion, I attach great significance to combined visual-spectroscopic orbits. Yet there are very few cases, a dozen at best, which may be really called combined orbits. In most other cases, the radial velocity has not been followed sufficiently continually.

Dommanget: And particularly, not followed by the same observer, or at the same instrument. This is quite important in order to know the precise shape of the $R V$ curve. Sometimes the orbit orientation may be reversed due to the failure to reduce the observations to a common system.

Heintz: Many binaries are of middle or late spectral class, and have good lines. A variation of the order of $5 \mathrm{~km} / \mathrm{s}$ then is usually trustworthy.

Dommanget: This is not always sufficient. A $R V$ difference of, say, $10 \mathrm{~km} / \mathrm{s}$ may be predicted. In the case of single-lined stars ( $\Delta \mathrm{m}$ large), the observed variation of the primary is less than half the difference $A$ minus $B$. In double spectra ( $\Delta m$ small), on the other hand, a larger velocity is also needed in order to resolve the blend.

Heintz: I was speaking of observed, not predicted, $R V$ variations. We also need the epochs of the spectroscopic observations. They can usually be found from the references given in R. E. Wilson's catalogue, or by checking with the older Lick and Voûte catalogues.

Dommanget: I know that it is often difficult, and sometimes impossible, to get the epochs. Even Wilson has not all of them. Of course, it is usually possible to time an observation within ten or twenty years. But this may not suffice if the radial velocity changes significantly during that period. 